\title{
Deborah S. Jin
}

1968-2016

\section{Pioneer of ultracold quantum physics.}

$\mathrm{D}$ eborah Jin invented ways to study a state of matter created in the mid-1990s: gases of strongly interacting atoms, cooled to near absolute zero. Her visionary and methodical approach made it possible to use these ultracold gases as model systems to tease out the quantum principles that lead to behaviours in real materials, such as superconductivity.

Jin, who died of cancer on 15 September, aged only 47, was born in 1968 in Stanford, California. She grew up in Indian Harbour Beach, Florida; her father was a professor of physics at Florida Institute of Technology. Her mother and brother also trained as physicists. A studious child, Jin won many mathematics competitions in school.

Jin completed an undergraduate degree in physics at Princeton University in New Jersey in 1990. She earned her PhD in 1995 from the at the University of Chicago in Illinois under Thomas Rosenbaum, studying unconventional superconductors that were cooled to millikelvin temperatures using liquid helium and similar traditional cryogenic techniques. Jin probed how the exotic superconductivity in these materials reacted to pressure, stress and magnetic fields. During this period, she met her husband, John Bohn, also a physics graduate student at Chicago. In the years that followed, they collaborated on several studies on the collisions of quantum particles.

Next, Jin made the bold decision to change research areas. She moved to Boulder, Colorado, to do postdoctoral research with Eric Cornell at JILA, a joint institute between the National Institute of Standards and Technology (NIST) and the University of Colorado, previously known as the Joint Institute for Laboratory Astrophysics. She worked on materials created with a new set of techniques - quantum gases of atoms cooled with lasers to microkelvin temperatures and suspended in vacuum by magnetic fields.

Jin quickly made key contributions to this new field, including measurements of the heat capacity and the excitation spectra of a Bose-Einstein condensate, a quantum phase of matter that Cornell had created for the first time with a colleague in 1995 . Bose-Einstein condensates comprise bosons, particles that have either zero or integer values of spin, that are all in the same spin state. Remarkably, the behaviour of collections of quantum particles, whether in superfluids or neutron stars, is determined

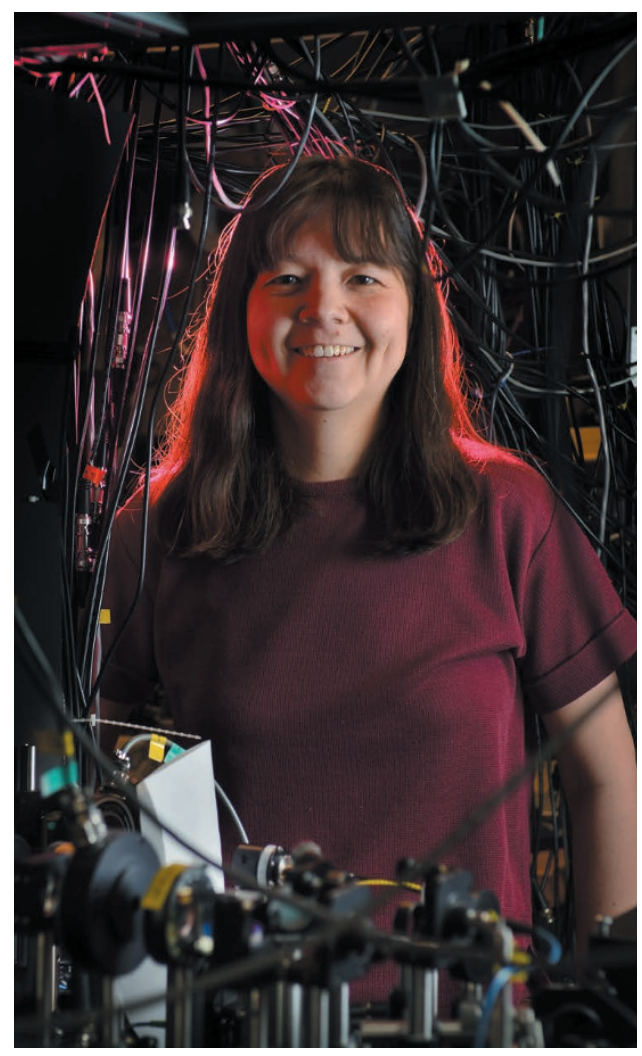

by whether they are made up of bosons or fermions, particles with half-integer spin.

In 1997, Jin accepted a permanent position at JILA and took on one of the greatest challenges in atomic physics at the time creating a gas of ultracold fermions. Only bosonic gases had been thus cooled when Jin chose to work with the fermionic isotope potassium-40. She realized that trapping two nuclear states of this rare atom was key to producing the first quantum Fermi gas, which her team did in 1999 (B. DeMarco and D. S. Jin Science 285, 1703-1706; 1999). The usefulness of these gases lies in their ability to emulate other models, for example of hightemperature superconductors.

The natural interactions between potassium-40 atoms are too weak to induce strong quantum correlations directly. So Jin and Bohn harnessed collisional resonances to strengthen the interaction between atoms using a magnetic field. They produced the first molecular Fermi condensate in 2003, and the first resonantly interacting Fermi gas in 2004.

Jin's work marked a shift in how other branches of physics viewed and interacted with experimental atomic physics. The early work on dilute atomic Bose-Einstein condensates could be understood using relatively simple and well-developed theoretical approaches. The achievement of strong interactions in the gas enabled experiments to connect with open and difficult physics questions, some of which still cannot be tackled using the most powerful supercomputers. This led to rich and varied exchanges between research areas, as exemplified by Jin's connections with nuclear and condensed-matter theorists.

In 2008, expanding her work from atoms, Jin partnered with Jun Ye at JILA to create the first quantum gas of diatomic molecules that experience long-range inter-particle interactions. These interactions lead to correlated behaviours in many-body quantum systems. The collaboration also helped to launch the study of chemical reactions in the ultracold quantum realm.

Despite a career cut tragically short, Jin's scientific legacy is broad and deep. Her research made textbook models a scientific reality, including ideal Fermi gases and the crossover between Bose-Einstein condensation and the Bardeen-Cooper-Schrieffer theory of superconductivity. She developed the tools and techniques to tune these states and conduct precise measurements, unveiling new physics along the way.

Debbie inspired a generation of young scientists who founded careers on the research directions she started. She was a warm, dedicated mentor and role model, and a champion of female physicists. She cared deeply about her students, colleagues and friends. Her laser-like focus and intellectual integrity could at times seem blunt, but her ex-students still ask themselves how Debbie would approach a problem.

Debbie loved living in Boulder and exploring the world with her husband and daughter, Jaclyn, who was often to be seen in the background at scientific meetings. Her bright smile will be missed by the many people whose lives she touched.

Brian DeMarco is a professor of physics at the University of Illinois UrbanaChampaign, Urbana, Illinois, USA. He was Debbie's first graduate student and worked with her on creating the first Fermi gas. John Bohn, Debbie's husband, and Eric Cornell, a collaborator, are fellows at JILA. e-mail: bdemarco@illinois.edu 